\title{
Leadership-Food - 10 Gebote für effektive und führungstaugliche Ernährung
}


Cynthia Ahrens • Leif Ahrens

\section{Leadership-Food - \\ 10 Gebote für effektive und führungstaugliche Ernährung}

Springer Gabler 
Cynthia Ahrens

Leif Ahrens

Wiesbaden

Deutschland

Die Deutsche Nationalbibliothek verzeichnet diese Publikation in der Deutschen Nationalbibliografie; detaillierte bibliografische Daten sind im Internet über http://dnb.d-nb.de abrufbar.

\section{Springer Gabler}

(C) Springer Fachmedien Wiesbaden 2014

Das Werk einschließlich aller seiner Teile ist urheberrechtlich geschützt. Jede Verwertung, die nicht ausdrücklich vom Urheberrechtsgesetz zugelassen ist, bedarf der vorherigen $\mathrm{Zu}$ stimmung des Verlags. Das gilt insbesondere für Vervielfältigungen, Bearbeitungen, Übersetzungen, Mikroverfilmungen und die Einspeicherung und Verarbeitung in elektronischen Systemen.

Die Wiedergabe von Gebrauchsnamen, Handelsnamen, Warenbezeichnungen usw. in diesem Werk berechtigt auch ohne besondere Kennzeichnung nicht zu der Annahme, dass solche Namen im Sinne der Warenzeichen- und Markenschutz-Gesetzgebung als frei zu betrachten wären und daher von jedermann benutzt werden dürften.

Gedruckt auf säurefreiem und chlorfrei gebleichtem Papier

Springer Gabler ist eine Marke von Springer DE. Springer DE ist Teil der Fachverlagsgruppe Springer Science+Business Media

www.springer-gabler.de 


\section{Ein paar Worte zu Beginn...}

Noch nie war die Auswahl an Lebensmitteln so vielfältig, abwechslungsreich und exotisch wie heute.

Noch nie war unsere Entscheidungsfreiheit, wie wir uns ernähren wollen, so umfassend und nahezu grenzenlos.

Und noch nie waren die ernährungsbedingten Herausforderungen für Führungskräfte so groß, um ihren Alltag ohne gesundheitliche Einbußen und mit gutem Gefühl bewältigen zu können. Verunsicherung und Überforderung spielen hierbei oftmals eine große Rolle. Nicht selten führt falsches Ernährungsverhalten bei Führungskräften zu Folgekrankheiten, Übergewicht oder ganz einfach zu Unzufriedenheit, weil sich kein Wohlbefinden und keine Befriedigung nach dem Essen einstellen.

Wie, was und wo Leader essen - das ist für Führungskräfte, die Erfolg haben wollen, also elementar. In den meisten Fällen bestimmt ein stark strukturierter Tagesablauf den Alltag von Menschen, die als Unternehmer oder in gehobenen Positionen arbeiten. Dies führt häufig dazu, dass Essen zur Nebensache wird. Entweder wird das Thema komplett in den Hintergrund gedrängt, so dass am Tag wenig und abends zu üppig getafelt wird, oder es gibt Tage, an denen eventuell sogar zwei Geschäftsessen anstehen. Die Menge an Lebensmitteln führt auch nicht zu einem guten Gefühl. Und der Alkohol tut sein Übriges.

Es ist jedoch gar nicht so schwer, sich durch ein paar Gedanken mit seiner Ernährung zu beschäftigen und diese auf den jeweiligen Arbeitsalltag anzupassen. Ohne große Einschränkungen oder Aufwand. Es ist einfach eine Überlegung im Vorfeld, wie und was gegessen werden soll. Das Wichtigste hierbei ist - wie bei allem im Leben - dass man daran Spaß hat und es nicht zu einer lästigen Sache wird. Essen ist etwas Schönes, das Energie spendet und glücklich machen kann. Die luxemburgische Spitzenköchin Lea Linster bringt es auf den Punkt, wenn sie sagt: „Leute, die schlecht essen - wie sollen die gut denken?!“ 
Wenn sich bereits Gewohnheiten eingeschlichen haben, die einer ausgewogenen Ernährung im Wege stehen, so können diese behutsam aufgegeben und durch bessere ersetzt werden. $\mathrm{Zu}$ vermeiden sind jedoch radikale Umstellungen, wozu auch Diäten gehören. Diese sind in der Regel kontraproduktiv, da die neuen Verhaltensweisen und Essensrituale nicht natürlich in den Alltag eingebaut bzw. dort umgesetzt werden können, sondern aufgezwungen sind.

Strikte Regeln, die in Büchern, Medien oder wissenschaftlichen Aufsätzen als unumgänglich aufgestellt werden, sind mit gesundem Menschenverstand zu betrachten, denn nicht jeder Mensch ist gleich gestrickt, was seinen Organismus und die benötigten Lebensmittel angeht. Es gilt natürlich, ein paar Grundsätze in Sachen Nährstoffe, Vitamine, Mineralien etc. zu befolgen, aber damit hat es sich auch schon, was allgemeine „Vorschriften“ angeht. Jeder muss für sich herausfinden, was er für das reibungslose Funktionieren von Körper und Geist benötigt.

Dies ist auch gar nicht so schwer. Probieren geht über Studieren. Und sich selbst zu beobachten und aufmerksam darauf zu achten, was guttut und was eher schadet, ist schon der erste Schritt zum Erfolg.

„Leadership-Food - 10 Gebote für effektive und führungstaugliche Ernährung“ zeigt in den drei großen Bereichen WIE, WAS und WO Leader essen einige Wege auf, die zu einer genussreichen und zugleich wirkungsvollen Ernährungsweise führen können. Dabei sind alle Gebote als Angebote zu verstehen, die für jeden in unterschiedlicher Weise zusammengesetzt werden können. Die einzelnen Unterpunkte vermitteln Grundwissen, um das eigene Ernährungsverhalten besser steuern und bestimmen zu können.

Viel Spaß beim Entdecken eigener Vorlieben und wohltuender Essensrituale!

Ihre Cynthia und Leif Ahrens 


\section{„Betriebsanleitung“" für Leadership- Food}

Mit dem vorliegenden Ernährungsbuch wollen wir Anregungen geben, wie Leader gut und nachhaltig essen können, so dass sie noch viele Jahre gesund und unbeschwert in ihrer Führungsaufgabe aufgehen können. Dabei werden den Lesern wissenschaftliche Brocken erspart, um das Lesen angenehm und leicht verständlich $\mathrm{zu}$ machen.

Das Buch besteht im Wesentlichen aus möglichst einfach umzusetzenden Tipps und Hinweisen, die nicht überfordern, gleichwohl ein gutes Maß an Interesse an der eigenen Ernährungsweise voraussetzen. Die Gebote appellieren an den gesunden Menschenverstand und sind nicht als zeit- und energieraubende Pläne zu verstehen, die das ganze Ernährungsverhalten radikal umzustellen versuchen. Anregungen und Impulse sollen zu einem langsamen und bedachtsamen Verändern einzelner Verhaltensweisen anregen, neugierig machen und im Ergebnis einen positiven Effekt erkennen lassen. Dies regt auch zu weiterem Erkenntnisgewinn an, der in den einzelnen Themenblöcken erlangt werden kann.

Drei große Themen werden angesprochen:

„WIE Leader essen“ befasst sich mit genussvollem, gesundem und achtsamem Ernährungsverhalten. Hierbei werden wir darauf eingehen, dass Essen Spaß machen soll, auch Kleinigkeiten wertgeschätzt werden können und das Gefühl nach dem Essen stimmen muss. Die Faktoren Stress und Langeweile können ebenfalls eine Rolle spielen, die das natürliche Hungergefühl überlagern und zu falschen Handlungsweisen führen.

„WAS Leader essen“ beschäftigt sich damit, die eigene Essenspalette möglichst vielseitig, individuell sowie regional und saisonal zu gestalten. Dort thematisieren wir buntes, vielfältiges und nährstoffreiches Essen sowie individuelles Essverhalten. Die Tatsache, dass jeder einen anderen Geschmack und bestimmte Vorlieben hat, wird hierbei gleichermaßen berücksichtigt. Weniger „allgemein gültige“ Anweisungen lautet hier das Motto, vielmehr soll das Beachten eigener Präferenzen in den Vordergrund gestellt werden. 
„WO Leader essen“ hat die unterschiedlichen Orte zum Inhalt, an denen Führungskräfte sich in der Regel aufhalten. So beschäftigen sich die hier behandelten Gebote mit der Ernährung im Büro, unterwegs auf Geschäftsreisen und last but not least zu Hause. Kantinenessen, Snacks bei Meetings sowie Restaurantbesuche spielen ebenso eine große Rolle, die wir näher beleuchten wollen.

Nach den drei großen Themenbereichen, die jeweils drei Gebote umfassen, wird in einem letzten (zehnten) Gebot umrissen, wie ein Foodmaster seine Ernährung gestalten kann. Selbstbestimmt essen, das Wissen, was guttut, und die Ablehnung ungeeigneter bzw. sogar schädlicher Ernährungsangebote sind hier das Thema. Nach den 10 Geboten kann ein kurzer Fragebogen mit 25 Fragen zur Reflexion beantwortet werden, um zu sehen, inwieweit es um die Aspekte "Wie“, „Was“ und „Wo“ in punkto Ernährung bestellt ist. Die Ergebnisse dieser Selbstreflexion münden im Leadership-Food-IQ, der das eigene Essverhalten skizziert. Ob noch an einigen Stellschrauben gedreht werden sollte, ergibt sich aus dem Endergebnis, das den Status auf dem Weg zum Foodmaster erkennen lässt.

Dieses Buch kann immer wieder zur Hand genommen werden, wenn sich schlechte Ernährungsgewohnheiten (wieder) einstellen, so dass mit ein paar Hinweisen und Tipps gegengesteuert werden kann. Das macht umso mehr Spaß, wenn ein positiver Effekt bemerkt wird, und regt zu weiteren (kleinen) Umstellungen an, die nach und nach zu einer tiefgreifenden Veränderung im Ernährungsverhalten führen können. Die Belohnung zeigt sich in mehr Energie, Tatkraft und Lebensfreude, welche zu einem Gutteil auf einer selbstbestimmten Ernährung beruhen. Dieses zu erkennen und umzusetzen, ist Ziel des Buches und Wunsch der Autoren für ihre Leser. 


\section{Inhaltsverzeichnis}

\section{Teil I WIE Leader essen}

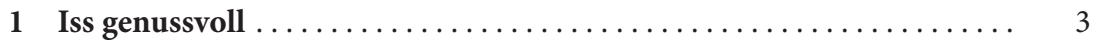

1.1 Spaß beim Essen haben .......................... 5

1.2 Auch kleine Mahlzeiten zelebrieren $\ldots \ldots \ldots \ldots \ldots \ldots \ldots \ldots . \ldots$

1.3 Mit anderen essen ist angenehmer als allein $\ldots \ldots \ldots \ldots \ldots \ldots . \ldots$

1.4 Mach' es Dir schön beim Essen ...................... 10

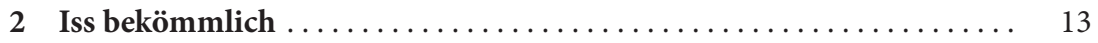

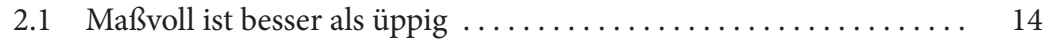

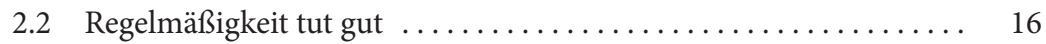

2.3 Wie ist das Gefühl nach dem Essen $\ldots \ldots \ldots \ldots \ldots \ldots \ldots \ldots . \ldots \ldots$

2.4 Lebensmittel, die den Einzelnen belasten können $\ldots \ldots \ldots \ldots \ldots \quad 20$

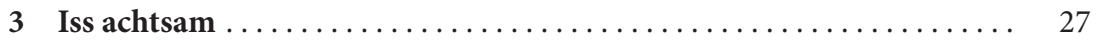

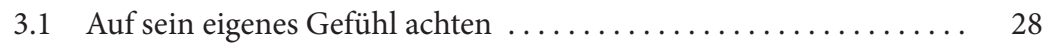

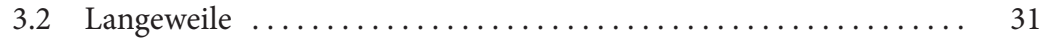

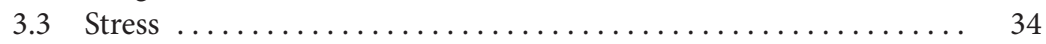

\section{Teil II WAS Leader essen}

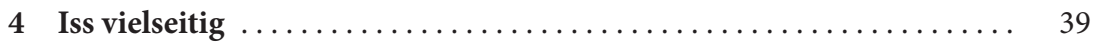

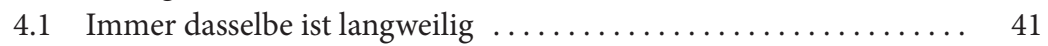

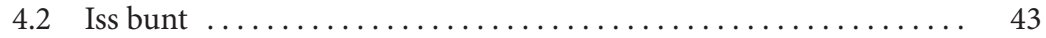

4.3 Grundnährstoffe, Vitamine und Co. - was ist das eigentlich? .... 45

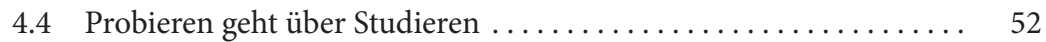




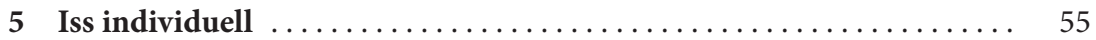

5.1 Jeder tickt bei der Ernährung anders $\ldots \ldots \ldots \ldots \ldots \ldots \ldots \ldots \ldots$

5.2 Geschmack und Vorlieben zählen .................... 58

5.3 Natürliche und wirkungsvolle „Notfallmaßnahmen“ $\ldots \ldots \ldots \ldots .60$

5.4 Andere Länder, andere Sitten ........................ 64

Literatur ........................................... 67

6 Iss regional und saisonal $\ldots \ldots \ldots \ldots \ldots \ldots \ldots \ldots \ldots \ldots \ldots \ldots \ldots$

6.1 Das Beste ist vor der Haustür . . . . . . . . . . . . . . . . . 70

6.2 Immer anders - das ganze Jahr über .................. 74

6.3 Im Vorfeld planen, im Nachhinein profitieren $\ldots \ldots \ldots \ldots \ldots \ldots \quad 77$

Teil III WO Leader essen

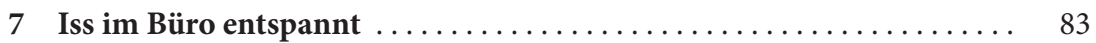

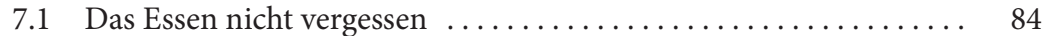

7.2 Kantinenessen und seine Tücken $\ldots \ldots \ldots \ldots \ldots \ldots \ldots \ldots \ldots$

7.3 Verführerische Meetings ....................... 88

7.4 Selbstmitgebrachtes - die Alternative zu Kantine und Schnellimbiss .............................. 89

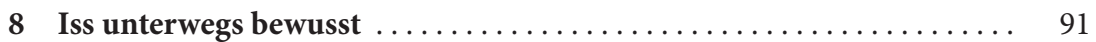

8.1 (Ernährungs-)Fallen auf Messen ................... 92

8.2 Restaurantbesuche ohne Nebenwirkungen $\ldots \ldots \ldots \ldots \ldots \ldots . . \ldots 4$

8.3 Bei Einladungen nicht alles „schlucken“ ............... 99

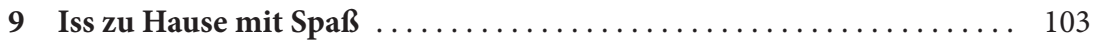

9.1 Der Einkauf entscheidet ........................ 104

9.2 Wer selbst kocht, bekommt immer sein Lieblingsgericht ....... 109

9.3 Mit Freunden gutes Essen genießen .................. 113

9.4 Einfach lecker essen...mit Rezepten von Merkel \& Co. ......... 115

Teil IV Finale: Wie, was und wo wir essen, macht den Erfolg aus

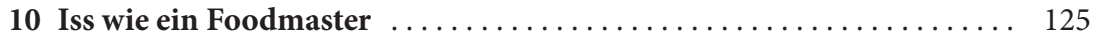

10.1 Selbstbestimmt essen .......................... 126

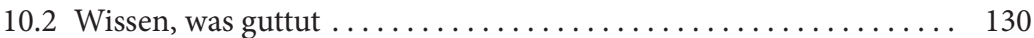

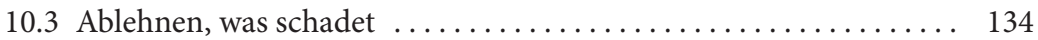




\section{Über die Autoren}

Cynthia und Leif Ahrens arbeiten seit vielen Jahren mit ihren Kunden in Vortragssälen, Seminar- und Coachingräumen sowie auf Bühnen zusammen.

Leif Ahrens (Dipl.-Medienwirt) hat 500 Interviews und $7000 \mathrm{~h}$ live auf Sendung bei einem der erfolgreichsten Privatsender Deutschlands (Hitradio FFH) absolviert, die eine solide Basis für Vorträge und Beratungen bilden. Im Plenum oder Einzelgespräch - wichtig ist für ihn, dass alle Impulse bekommen und Ansätze finden, erfolgreicher und präsenter zu sein.

Cynthia Ahrens (M.A. Sinologie) optimierte als PR-Beraterin viele Jahre den Auftritt von Führungskräften in der Öffentlichkeit. Durch Beobachtung und Beratung wurde die Entwicklung von Persönlichkeiten zu ihrem Alltagsgeschäft. Nach Psychologie-Weiterbildung und Zertifizierung zur "Persönlichkeits-Profilerin“ arbeitet sie heute mit Führungskräften und verarbeitet dieses Wissen in Büchern und auf Portalen. Ihre Ausbildung zur Ernährungsberaterin und Phytotherapeutin schürte die Motivation, ein Ernährungsbuch speziell für Führungskräfte zu entwickeln. 


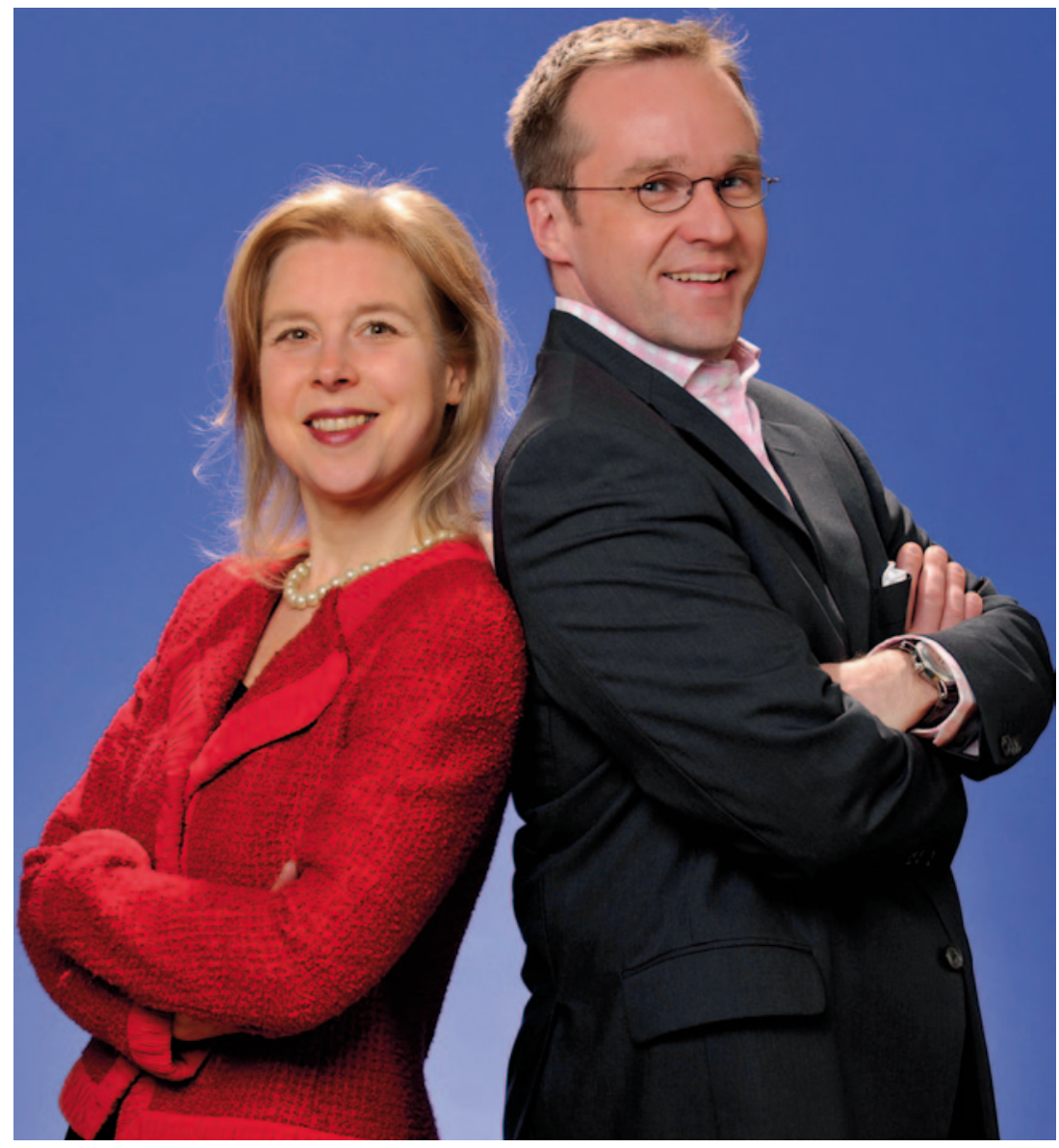

\section{Buchung:}

Anfragen für Vorträge, Seminare und Coachings von Cynthia und Leif Ahrens zum Thema Leadership-Intelligenz an info@leifahrens.de. 